\title{
Inadvisable Treatment of Recalcitrant Septic Olecranon Bursitis: An Unusual Case of Extremely Prolonged Treatment With Oral Antibiotics After Bursectomy
}

\author{
Todd C. Pitts ${ }^{\mathrm{a}}$, Casey J. Kiser ${ }^{\mathrm{a}}$, John G. Skedros ${ }^{\mathrm{a}, \mathrm{b}}$
}

\begin{abstract}
The intention of this report is to alert physicians about how prolonged the course of treatment for recalcitrant septic olecranon bursitis (OB) might be when only oral antibiotics are used. Some patients may need extra convincing that this course of treatment could be so long that it would not be recommended (i.e., a relatively shorter course of i.v. antibiotics is preferred). We treated a 56-yearold healthy male who developed methicillin-sensitive Staphylococcus aureus (MSSA) OB after a scorpion sting. The infection was not eradicated with bursectomy and a conventional course of antibiotics. The unusual persistence of the infection was initially attributed to venom, but olecranon osteomyelitis was eventually suspected. The patient refused a typical and predictably effective course of i.v. antibiotics because this would have constrained his business travel schedule. Consequently, full resolution required 240 days; treatment being mainly with two concurrent antibiotics (trimethoprim/sulfamethoxazole and rifampin).
\end{abstract}

Keywords: Olecranon bursitis; Olecranon osteomyelitis; Elbow infection; Oral antibiotics; Patient compliance

\section{Introduction}

Although septic olecranon bursitis (OB) usually resolves

Manuscript accepted for publication December 7, 2011

${ }^{a}$ Utah Bone and Joint Center, Salt Lake City, Utah, U.S.A

${ }^{\mathrm{b}}$ Corresponding author:John G. Skedros, 5323 South Woodrow Street, Suite 200, Salt Lake City, Utah 84107, U.S.A.

Email: jskedros@utahboneandjoint.com uneventfully with relatively short courses of oral or intravenous (i.v.) antibiotics, some cases with virulent or persistent infections might require increased durations of i.v. antibiotics in addition to surgical bursectomy [1-4]. However, even in cases of recalcitrant infections there can be situations where patient demands or clinical circumstances might compel physicians and other healthcare providers to attempt to eradicate an infection with the use of oral antibiotics alone. In our clinic we occasionally see patients who are referred for potential additional surgical or medical management of persistent septic OB. In these cases initial bursectomy and antibiotics have failed to eradicate the infection, and continuing treatment with oral antibiotics seems to have only a suppressive effect even though they have typically been taken for over six weeks. Explanations for the persistence of these infections include the resistant or atypical organisms that fail to grow in culture, poor tissue penetration of the oral antibiotic, and/or the development of olecranon osteomyelitis. Currently, the published literature is sparse with respect to the potential duration of treatment that might be required when special circumstances necessitate consideration of treatment with only oral (and seemingly appropriate) antibiotics for atypical cases where the infection persists despite a bursectomy and an initial conventional course of antibiotics.

In the perspective of these referred cases of unusually recalcitrant septic $\mathrm{OB}$, we could not locate any reports that provide information for decision making and patient education regarding the potential duration of oral, when compared to typical i.v., antibiotic treatment that might be required to eradicate the infection. More specifically, we could not locate information that would help in estimating the potential duration of the oral-only antibiotic treatment that is requested by some patients (i.e., those that refuse i.v. antibiotics) including the patient that we describe here.

We describe a case of septic OB resulting from a scorpion sting that, despite initial bursectomy, organism-appropriate i.v. antibiotics, and subsequent multiple irrigations and debridements, took nearly 240 days of two concurrent oral antibiotics for full resolution. One important reason for this prolonged duration for complete resolution was not because of tissue necrosis, but because the patient refused i.v. antibiotics in order to avoid impeding his demanding business and 


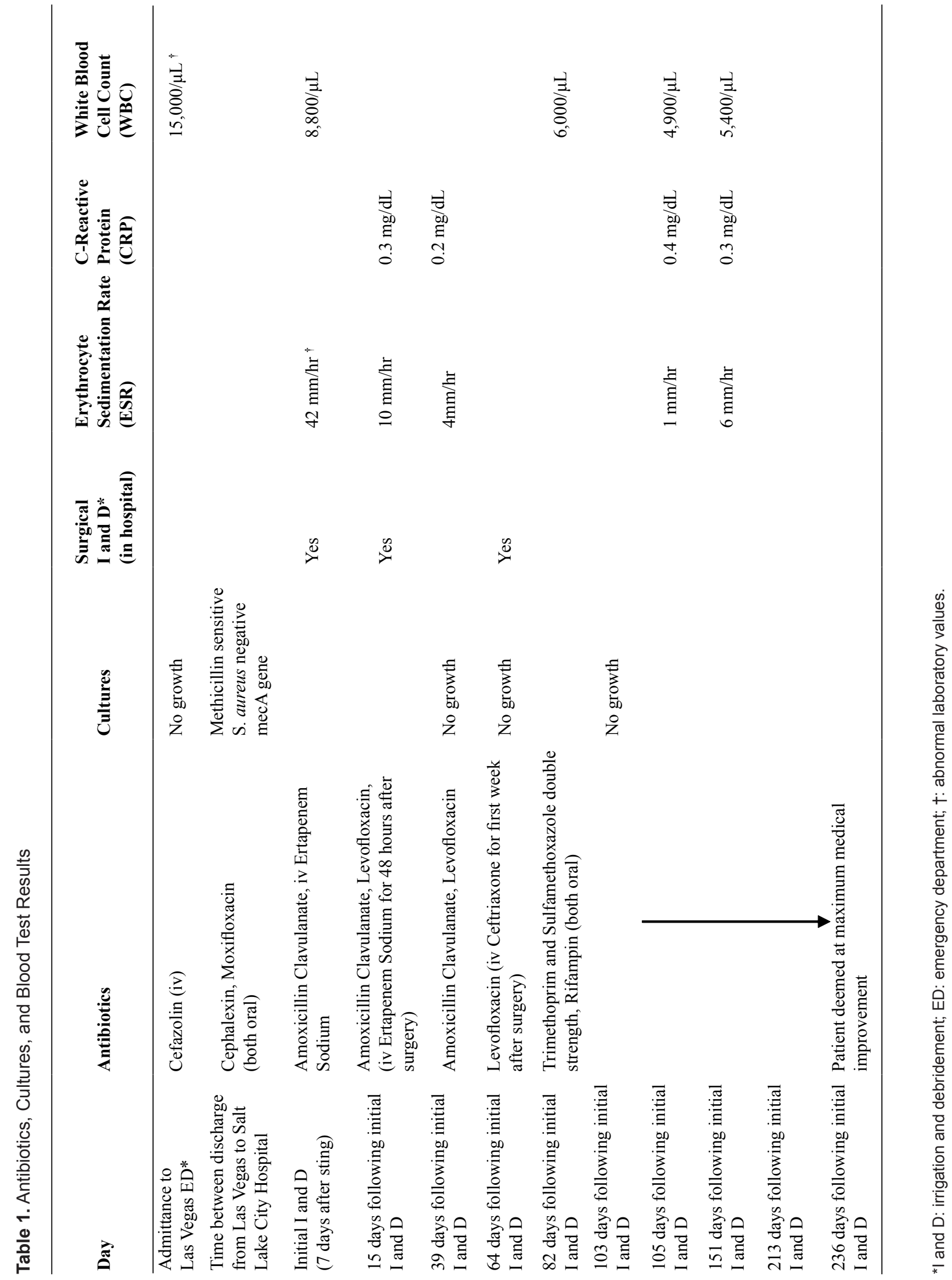




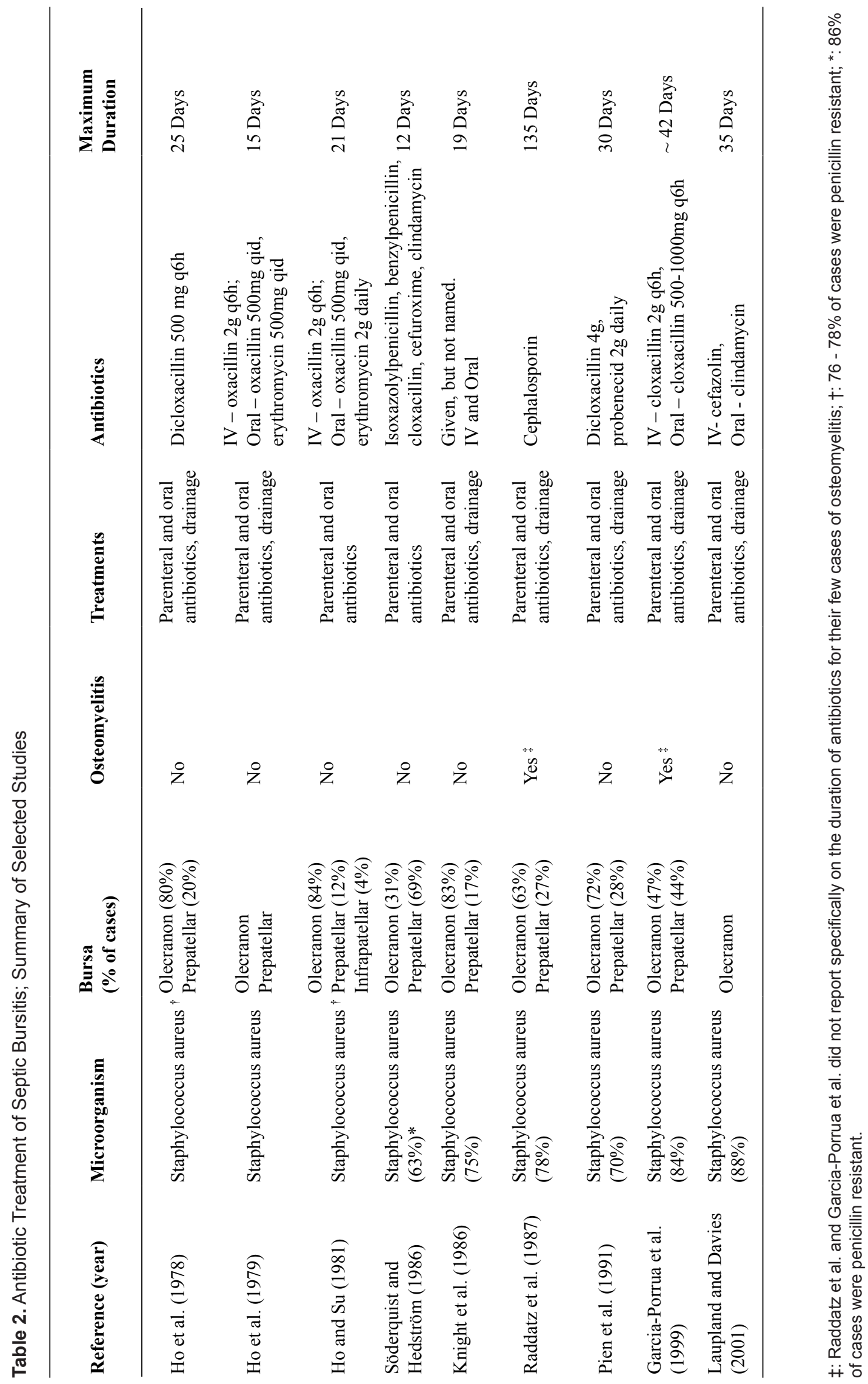



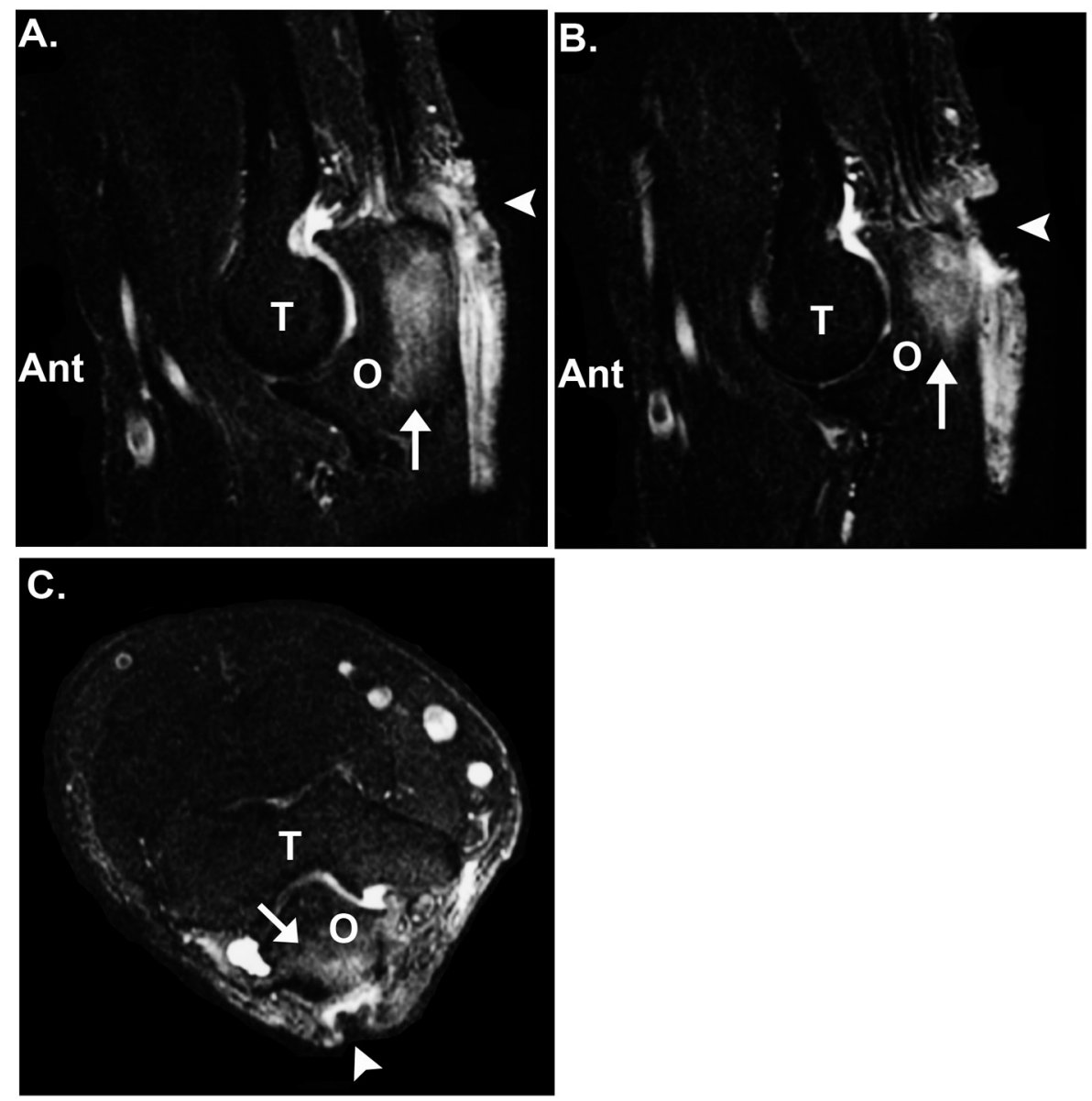

Figure 1. Lateral (A, B) and axial (C) MRI views of the patient's right elbow. Signal changes are seen in the posterior portion of the olecranon $(\mathrm{O})$ process (arrow) and adjacent periosteal and subcutaneous tissue. Additionally, the open wound is seen on the posterior aspect of the elbow (arrow head). T: trochlea of humerus; Ant.: anterior in images A and B.

personal travel schedule. An additional important aspect of this case is that underlying olecranon osteomyelitis was suspected, which helps to explain why a long duration of two oral antibiotics was required.

We report this case to illustrate how the duration of successful oral-only antibiotic treatment can be extremely prolonged, and hence is typically inadvisable, for some cases of recalcitrant septic OB.

\section{Case Report}

A 56-year-old Caucasian male, who worked as an engineer, presented to an emergency department in Las Vegas, Nevada, USA with pain, swelling, and erythema on the posterior aspect of his right elbow. He was $188 \mathrm{~cm}$ tall, weighed 110 $\mathrm{kg}\left(\mathrm{BMI} 31 \mathrm{~kg} / \mathrm{m}^{2}\right.$ ), and reported being in excellent health. His past medical history corroborated that he was not taking medications, did not smoke, occasionally drank alcohol, and had no personal or family history of heart/vascular disease, diabetes, autoimmune disorder, or poor wound healing.
On the previous morning, he was working in the desert near Henderson, Nevada. While clearing sagebrush from beneath his truck, he felt a sharp stinging sensation on his right elbow as it contacted the ground. He thought that a scorpion might have stung him, but he did not attempt to retrieve the organism. The following day he was admitted to a cardiac monitoring unit of a local hospital because of a ventricular arrhythmia (premature ventricular contractions), tachycardia, and hypertension (177/104 mmHg). A twelve-lead electrocardiogram showed multiple pre-ventricular contractions and nonspecific S-T wave changes, but no infarction pattern. It was surmised that these abnormalities were caused by envenomation $[5,6]$. Punctate erythematous marks at the site of injury were consistent with the sting of a bark scorpion (Centruroides exilicauda), which explained the arrhythmia and hypertension within 24 hours of the sting [7, 8]. Blood tests showed normal cardiac enzymes and elevated glucose (137 mg/dL, normal 65 - $99 \mathrm{mg} / \mathrm{dL}$ ). A complete blood count $(\mathrm{CBC})$ revealed leukocytosis $(15,000 / \mu \mathrm{L}$ ) (normal 4,000 $10,500 / \mu \mathrm{L})$ and a low platelet count $(105 / \mu \mathrm{L}$, normal 150 $-450 / \mu \mathrm{L}$ ). Blood cultures revealed no growth. The cellulitis 
was treated with i.v. cefazolin.

An adenosine cardiac stress test revealed reduced ejection fraction (46\%; normal $50-75 \%)$ and mild decreased tracer uptake in the inferior wall on the stress/rest studies. Ventricular cavity size was within normal limits. In view of these relatively minor findings, and the rapid improvement of the hypertension and arrhythmia with $50 \mathrm{mg}$ /day of oral metoprolol tartrate, further work-up was not conducted. One day later, he was discharged to his home with metoprolol tartrate $50 \mathrm{mg}$ /day and two oral antibiotics: cephalexin $500 \mathrm{mg}$ four times a day and moxifloxacin hydrochloride $400 \mathrm{mg}$ / day.

At admission for bursectomy in Salt Lake City, Utah, his erythrocyte sedimentation rate (ESR) was elevated at 42 $\mathrm{mm} / \mathrm{hr}$ (normal $0-20 \mathrm{~mm} / \mathrm{hr}$ ). Wound cultures from the bursectomy grew methicillin-sensitive Staphylococcus aureus (MSSA) (Table 1). Treatment included one gram of i.v. ertapenem sodium for three days in the morning followed by two weeks of oral $875 \mathrm{mg}$ of amoxicillin/clavulanate potassium twice a day, and daily dressing changes for the open wound.

At two weeks after surgery, his ESR and C-reactive protein (CRP) were normal and primary closure was done. One week later, purulent drainage recurred. Repeated bacterial, fungal, and tuberculosis cultures showed no growth (Table 1). Another irrigation/debridement was done and the wound was left open. The patient continued taking $500 \mathrm{mg}$ of oral levofloxacin each morning and $875 \mathrm{mg}$ of oral amoxicillin/ clavulanate potassium every evening, and wound irrigations and dressing changes were done in the clinic. The patient refused our recommendation that i.v. antibiotics should be used for treatment, stating that allowing this would preclude his demanding business and personal travel schedule.

An infectious disease specialist recommended switching antibiotics to the concurrent use of oral double-strength trimethoprim/sulfamethoxazole two tablets three times a day (t.i.d.) and oral rifampin $300 \mathrm{mg}$ t.i.d. for broad bacterial coverage and good bone penetration for the possibility of underlying osteomyelitis. Continued use of oral-only antibiotic treatment reflected the patient's insistence on avoiding i.v. treatment. Aerobic and anaerobic cultures obtained at 64 and 103 days after the initial bursectomy continued to show no growth (Table 1). ESR and CRP values also remained within normal limits. Although the magnetic resonance (MR) scans that were then obtained showed findings consistent with osteomyelitis (Fig. 1), continuing oral antibiotics with this double regimen was considered reasonable because of progressive improvement including: (1) retraction of the wound margins, (2) blood tests remaining within normal ranges, and (3) no evidence of systemic toxicity. Treatment now also included warm whirlpool treatments to enhance vascularization.

By 200 days after initial bursectomy, the diameter of the wound had contracted to one millimeter. The infection was considered eradicated by 236 days following the initial bursectomy. Four years later the patient remained free of recurrence and had no deficits in elbow function. Although he was extremely pleased with the final outcome, he stated that in retrospect he would have consented to i.v. antibiotic treatment had he realized that nearly eight months of oral-only treatment might be required.

\section{Discussion}

Among all bursae, sepsis most commonly occurs in the olecranon bursa $[2,4,9,10]$, with arthropod bites or stings being a frequent cause. Staphylococcus aureus is the cause in over $80 \%$ of cases $[3,4,9]$. One explanation for the persistence of our patient's infection is the possibility that the wound was also inoculated with an atypical organism (e.g., Nocardia or atypical mycobacterium) that was never identified. Regardless of the possible cause of our case of septic OB, it is distinctive in the reported literature because of the extremely long duration of oral antibiotic treatment (Table 2) [1-4, 1014]. Raddatz et al. [10] is the only report that we could find of prolonged oral treatment, with a range of $10-135$ days (mean 40 days).

Osteomyelitis seems to be the best explanation for the persistence of the infection. Although successful treatment of acute osteomyelitis with long-term oral antibiotics is a well established form of treatment $[15,16]$ and successful treatment of recalcitrant or virulent bacterial infections with oral trimethoprim/sulfamethoxazole alone or concurrently with rifampin have been described [17-20], we could not locate a case of septic OB (with or without olecranon osteomyelitis) that required using these two antibiotics with prolonged duration similar to that required for our patient. Scully and co-workers [21] reported on the prolonged use (mean 40 days, range: 5 - 283 days) of oral ofloxacin for infections due to multiple-resistant bacteria, including Staphylococcus aureus. However, in that study there was no report of septic $\mathrm{OB}$ and the prolonged treatments were for osteomyelitis or infections associated with endoprostheses.

Similar to our case report, none of the 82 patients in the previous report of Scully et al. [21] had hepatic, renal, or hematologic toxicity despite long therapy. Additional complications that can result from long-term oral antibiotics include Clostridium difficile diarrhea and antibiotic-related marrow suppression (e.g., thrombocytopenia). Because of these and other potential complications, especially the length of prolonged therapy and potential increased cost, we do not advocate treatment using a prolonged course of oral antibiotics instead of conventional parenteral antibiotics for exceptional cases of virulent/persistent septic OB that may or may not be associated with olecranon osteomyelitis. A drug holiday could have been suggested with repeat cultures in order to better elucidate the target organism or to simply confirm bac- 
terial eradication without underlying antibiotic serum levels.

In conclusion, this report shows how prolonged the course of treatment of recalcitrant septic olecranon bursitis (OB)/osteomyelitis can be when using only oral antibiotics. This report should help healthcare providers to educate some patients about the wisdom and comparative benefits of accepting i.v. antibiotic treatment when they might otherwise refuse it.

\section{Conflict of Interest}

The authors have no conflicts of interest in either a financial or other capacity that would bias this work.

\section{References}

1. Garcia-Porrua C, Gonzalez-Gay MA, Ibanez D, GarciaPais MJ. The clinical spectrum of severe septic bursitis in northwestern Spain: a 10 year study. The Journal of rheumatology. 1999;26(3):663-667.

2. Ho G, Jr., Su EY. Antibiotic therapy of septic bursitis. Its implication in the treatment of septic arthritis. Arthritis and rheumatism. 1981;24(7):905-911.

3. Laupland KB, Davies HD. Olecranon septic bursitis managed in an ambulatory setting. The Calgary Home Parenteral Therapy Program Study Group. Clinical and investigative medicine. Medecine clinique et experimentale. 2001;24(4):171-178.

4. Ho G, Jr., Tice AD, Kaplan SR. Septic bursitis in the prepatellar and olecranon bursae: an analysis of 25 cases. Annals of internal medicine. 1978;89(1):21-27.

5. Santhanakrishnan BR, Gajalakshmi BS. Pathogenesis of cardiovascular complications in children following scorpion envenoming. Annals of tropical paediatrics. 1986;6(2):117-121.

6. Yarom R, Gueron M, Braun K. Scorpion venom cardiomyopathy. Pathologia et microbiologia. 1970;35(1):114117.

7. Carbonaro PA, Janniger CK, Schwartz RA. Scorpion sting reactions. Cutis; cutaneous medicine for the practitioner. 1996;57(3):139-141.

8. Curry SC, Vance MV, Ryan PJ, Kunkel DB, Northey WT. Envenomation by the scorpion Centruroides sculpturatus. Journal of toxicology. Clinical toxicology. 1983;21(4-5):417-449.

9. Cea-Pereiro JC, Garcia-Meijide J, Mera-Varela A, Gomez-Reino JJ. A comparison between septic bursitis caused by Staphylococcus aureus and those caused by other organisms. Clin Rheumatol. 2001;20:10-14.
10. Raddatz DA, Hoffman GS, Franck WA. Septic bursitis: presentation, treatment and prognosis. The Journal of rheumatology. 1987;14(6):1160-1163.

11. Ho G, Jr., Tice AD. Comparison of nonseptic and septic bursitis. Further observations on the treatment of septic bursitis. Archives of internal medicine. 1979;139(11):1269-1273.

12. Soderquist B, Hedstrom SA. Predisposing factors, bacteriology and antibiotic therapy in 35 cases of septic bursitis. Scandinavian journal of infectious diseases. 1986;18(4):305-311.

13. Knight JM, Thomas JC, Maurer RC. Treatment of septic olecranon and prepatellar bursitis with percutaneous placement of a suction-irrigation system. A report of 12 cases. Clinical orthopaedics and related research. 1986(206):90-93.

14. Pien FD, Ching D, Kim E. Septic bursitis: experience in a community practice. Orthopedics. 1991;14(9):981-984.

15. Daver NG, Shelburne SA, Atmar RL, Giordano TP, Stager CE, Reitman CA, White AC, Jr. Oral step-down therapy is comparable to intravenous therapy for Staphylococcus aureus osteomyelitis. The Journal of infection. 2007;54(6):539-544.

16. Shuford JA, Steckelberg JM. Role of oral antimicrobial therapy in the management of osteomyelitis. Current opinion in infectious diseases. 2003;16(6):515-519.

17. Marcotte AL, Trzeciak MA. Community-acquired methicillin-resistant Staphylococcus aureus: an emerging pathogen in orthopaedics. The Journal of the American Academy of Orthopaedic Surgeons. 2008;16(2):98-106.

18. Anstead GM, Quinones-Nazario G, Lewis JS, 2nd. Treatment of infections caused by resistant Staphylococcus aureus. Methods in molecular biology. 2007;391:227258.

19. Stryjewski ME, Chambers HF. Skin and soft-tissue infections caused by community-acquired methicillinresistant Staphylococcus aureus. Clinical infectious diseases : an official publication of the Infectious Diseases Society of America. 2008;46 Suppl 5:S368-377.

20. Walsh TJ, Standiford HC, Reboli AC, John JF, Mulligan ME, Ribner BS, Montgomerie JZ, et al. Randomized double-blinded trial of rifampin with either novobiocin or trimethoprim-sulfamethoxazole against methicillinresistant Staphylococcus aureus colonization: prevention of antimicrobial resistance and effect of host factors on outcome. Antimicrobial agents and chemotherapy. 1993;37(6):1334-1342.

21. Scully BE, Clynes N, Neu HC. Oral ofloxacin therapy of infections due to multiply-resistant bacteria. Diagnostic microbiology and infectious disease. 1991;14(5):435441 . 